\title{
Effect of Pulse Number on Dopant Activation in Silicon during Shallow p+/n Junction Formation by Non-Melt Excimer Laser Annealing
}

\author{
SitiRahmah Aid ${ }^{1, a^{*}}$ Azura Hamzah ${ }^{1, \mathrm{~b}}$, Sumiaty Ambran ${ }^{1, \mathrm{c}}$, Satoru Matsumoto ${ }^{1, \mathrm{~d}}$, Zaharah Johari $^{2, \mathrm{e}}$, and Genshu Fuse ${ }^{3, \mathrm{f}}$ \\ ${ }^{1}$ Malaysia-Japan International Institute of Technology, UniversitiTeknologi Malaysia, Jalan Sultan Yahya Petra, 54100 Kuala Lumpur, \\ Malaysia \\ ${ }^{2}$ Faculty of Electrical Engineering, UniversitiTeknologi Malaysia, 81310 Skudai, Johor, Malaysia \\ ${ }^{3}$ SEN Corporation, SBSTower 9F, 4-10-1 Yoga, Setagaya-ku, Tokyo 158-0097, Japan
}

\begin{abstract}
Shallow p+/njunctions were formed using Ge-PAI and low-energy/high-dose boron-ion implantation, followed by thermal annealing involving nanosecondnon-melt excimer laser annealingandrapidthermal annealing. The effect of laser pulse number on dopant activation and regrowth of a-Si layer are discussed.We found that laser annealing aloneis insufficient to regrow a-Si layer and activate dopant. In contrast, activationwas observed in the samples that were subjected to preannealingrapid thermal annealing followed by non-melt laser annealing; with slight increase of sheet resistance valuewhen the number of laser pulse shot was increased. This is considered due to the interaction of dopant and supersaturated defect in the remaining a-Si layer.The percentage of defect-dopant interaction increased with the pulse shot numbers which contributed to the decrease in activation level.
\end{abstract}

\section{Introduction}

The realization ofefficientand lower energy-consuming devices has been relied on the continuousdownscaling of silicon ( $\mathrm{Si}$ )-MOS transistor dimensions. However, the ongoing downscaling of planar silicon $(\mathrm{Si})$ has becoming more difficultdue to the physical limitation issues.One of the severe problems is the short channel effects (SCE) that occur when the channel length is shortened.This problem will reduce the device threshold voltage and subsequently increase the leakage current [1-3].

Formation of highly doped and activated shallow $\mathrm{p}^{+} / \mathrm{n}$ junction for source/drain extension (SDE) region is considered as one of the promising methods for suppressing this problem $[2,3]$. The simplest way for forming shallow junction in $\mathrm{Si}$ is by using the combination of the low-energy ion implantation and rapid thermal annealing. However, formation of the highly doped/activated and diffusion-less shallow $\mathrm{p}^{+} / \mathrm{n}$ junction has become an important challenge due to the boron ion channeling and anomalous boron diffusion problems [4].

Introducing germanium pre-amorphization (Ge-PAI) prior to the low-energy implantation is one of the promising methods to suppress the boron ion channeling problem. Low-energy boron-ion implantation can then be performed with high dose concentration to obtain highly doped of boron near surface [5-8].
Recently, non-melt laser annealing (NLA) process has been arise as one of the promising candidate to regrowamorphous silicon layer (a-Si) and activate dopant without meltingin an extremely short time $[9,10]$. This ultra-fast annealing time is important to prevent the anomalous dopant diffusion during annealing process. This paper reports the formation of shallow $\mathrm{p}^{+} /$njunctions using Ge-PAI and low-energy/high-dose boron-ion implantation, followed by thermal annealing involving nanosecondNLA andrapidthermal annealing (RTA). The effect of NLA pulse number on dopant activation and regrowth of a-Si layer are discussed.

\section{Experimental details}

(100) - oriented n-type Si substrates with resistivity of $10-\Omega \cdot \mathrm{cm}$ were used in this study. Ge-PAI with energy of $3-\mathrm{keV}, 3 \times 10^{14} \mathrm{~cm}^{-2}$ dose concentration and $7^{\circ}$ tilt angle was performed to produce an amorphous surface layer. Transport-of-Ions-in-Matter (TRIM) calculation was performed to calculate the amorphous layer created from Ge-PAI. Amorphous region was created by assuming that the displacement atom density has exceeded single-crystal's atomic density, i.e. $5 \times 10^{22}$ atom $/ \mathrm{cm}^{3}$ (see Fig. 1). The a-Si layer thickness calculated under these conditions is $\sim 5 \mathrm{~nm}$; which agrees well with the cross-sectional high-resolution transmission electron microscopy (X-TEM) analysis[7, 8]. Subsequently, low-energy boron-ion implantation

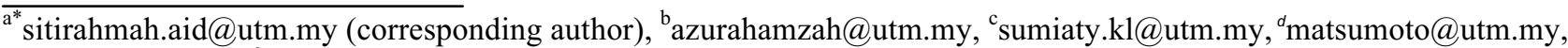
ezaharah@utm.my, fuse_gns@senova.co.jp
} 
was performed with energy of $0.3 \mathrm{keV}$, dose of $1.2 \times$ $10^{15} \mathrm{~cm}^{-2}$ and tilt angle of $0^{\circ}$. As-implanted secondary ion mass spectrometry (SIMS) profile of the sample is shown in Figure 2.

Implanted samples were then annealed using two different annealing conditions i.e. laser annealed-only and preannealing RTA followed by laser annealing. Laser annealing was performed using $\mathrm{KrF}$ excimer laser in a N2 ambient. The wavelength of the laser is $248 \mathrm{~nm}$ with beam shape of Gaussian distribution and pulse duration (full width at half maximum) of $32 \mathrm{~ns}$. The laser beam passed through a homogenizerand $0.5 \mathrm{~mm} \times$ $100 \mathrm{~mm}$ line beam was formed at a pulse repetition rate of $20 \mathrm{~Hz}$. Samples were annealed by scanning the line beam with a $98 \%$ overlap. Number of pulse shot was varied between $10-100$ shots to evaluate the effect of laser-pulse-number on activation. For NLA-only samples, laser energy densities were selected between $250-500 \mathrm{~mJ} / \mathrm{cm}^{2}$ in order to anneal without melting. For the RTA + NLA samples, laser energy was fixed at 300 $\mathrm{mJ} / \mathrm{cm}^{2}$. PreannealingRTAwas performed in $\mathrm{N}_{2}$ ambient at $450^{\circ} \mathrm{C}$ for $10-60 \mathrm{~s}$.

It is important to monitor the surface temperature during NLA to define the onset of melting. Onedimensional heat flow equation (Eq. 1) was numerically solved to determine the surface temperature. The calculation was performed using two layer model (i.e. a$\mathrm{Si}$ layer on $\mathrm{c}-\mathrm{Si}$ substrate).The detail of this calculation is described by Siti et.al. [7]

$$
\rho c\left(\frac{\partial T}{\partial t}\right)=\frac{\partial}{\partial x}\left(\lambda \frac{\partial T}{\partial x}\right)+\alpha(1-R) I_{0}(t) \exp (-\alpha x)(1)
$$

where $\mathrm{Q}$ is mass density, $\mathrm{c}$ is specific heat capacity, $\lambda$ is thermal conductivity, $\alpha$ is absorption coefficient and R is reflection coefficient.

The evaluation of surface morphology was performed using atomic force microscopy (AFM) to observe the surface roughness caused by melting. Single wavelength ellipsometry measurements (laser source: $\mathrm{He}-\mathrm{Ne}$ ) was performed to determine the refractive index of the regrowth layer after annealing. Dopant activation was evaluated from the sheet resistances $\left(R_{s}\right)$ that were obtained by Hall measurements.

\section{Results anddiscussion}

Table 1 shows the sample surface temperatures calculated using the numerical calculation of heat flow equation for the selected laser energy densities after single shot laser irradiation [7]. The energy density range for NLA is $300-400 \mathrm{~mJ} / \mathrm{cm}^{2}$ with the temperature range of $1078-1323 \mathrm{~K}$. The temperature exceeds the a$\mathrm{Si}$ melting temperature of $1420 \mathrm{~K}$ when the energy densities reach over $450 \mathrm{~mJ} / \mathrm{cm}^{2}$. This is also being confirmed by the AFM measurement. The surface roughness is observed when the samplewasannealed at $450 \mathrm{~mJ} / \mathrm{cm} 2$. Therefore, it can be concluded that the onset of melting is between $400-500 \mathrm{~mJ} / \mathrm{cm}^{2}$.

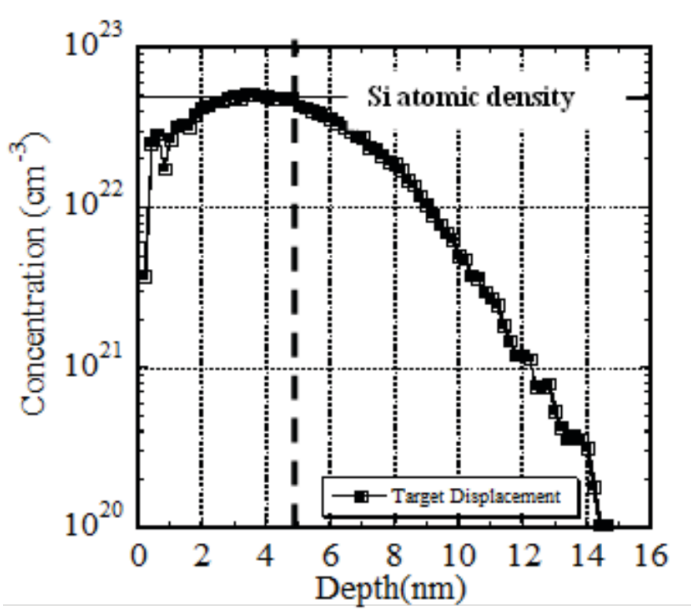

Figure 1.TRIM calculation for a-Si layer created in asimplanted sample with a Ge-PAI energy of $3 \mathrm{keV}$ and a dose of $3 \times 10^{14} \mathrm{~cm}^{-2}$

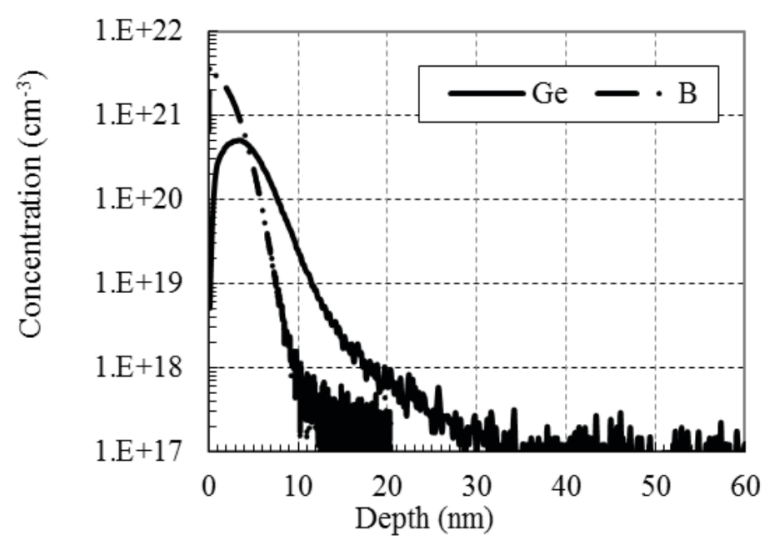

Figure 2. SIMS profiles of germanium and boron for asimplanted sample [7]

Table 1. Peak surface temperature and surface roughness for different laser energy densities

\begin{tabular}{|l|c|c|c|c|}
\hline $\begin{array}{l}\text { Laser energy density } \\
\left(\mathrm{mJ} / \mathrm{cm}^{2}\right)\end{array}$ & 300 & 350 & 400 & 450 \\
\hline Peak temperature $(\mathrm{K})$ & 1078 & 1205 & 1323 & 1420 \\
\hline Surface roughness $(\mathrm{nm})$ & \multicolumn{3}{|c|}{$\begin{array}{c}0.95 \text { (same as as- } \\
\text { implanted sample) }\end{array}$} & 1.89 \\
\hline
\end{tabular}

Figure 3 shows the Hall measurement results of the samples that were only laser annealed. The number of shots changed from 10 to 20 shots. High sheet resistance value is observed while the laser-pulse number dependence of activation is not observed in the samples that were annealed with 10 and 20 shots. It is considered due to the instability in the Hall measurements which only occur when large amount of a-Si layer was present. The remaining of a-Si layer is confirmed from the refractive index of the surface layer that was analyzed using single-wavelength ellipsometry measurements. As shown in Figure 4, the refractive index of the surface layer in the samples that were only laser annealed is still higher than the value of $\mathrm{c}-\mathrm{Si}$. Therefore, we may conclude that increasing the number 
of pulse shot number in the samples that were only laser annealed could not contribute to the improvement in activation. This is due to the NLA alone is insufficient to regrow of a-Si layer and dopant activation, as reported previously [7].

Figure 5 shows the sheet resistance of the samples subjected to preannealing RTA followed by NLA with number of laser pulse shot varied between $10-100$ shots. Stable Hall measurement could be performed in these samples implying that the regrowth of a-Si layer had begun and that boron was activated during annealing. It is observed that sheet resistance is slightly increased with number of laser pulse shot, except for the samples of $450^{\circ} \mathrm{C} 10 \mathrm{~s}$ RTA + NLA. This is considered as large part of a-Si layer still remains after the annealing process under this condition. Consequently, number of dopant incorporated into substitutional sites is lower than those of $30 \mathrm{~s}$ and $60 \mathrm{~s}$. However, the values for $R_{s}$ in all samples annealed for $10-60 \mathrm{~s}$ are still high as a-Si layer still present due to the insufficient recrystallization under these three RTA annealing conditions. The remaining of a-Si layer is saturated with defects that will interact with dopant during the subsequent NLA[11]. This interaction may results in the deactivation of dopant. The percentage of defect-dopant interaction increased with the pulse shot number which will contribute to the decrease in activation level. Therefore, we may assume that the optimum number of laser pulse shot to achieve higher activation level in the sample of RTA followed by NLA is 10 shots.

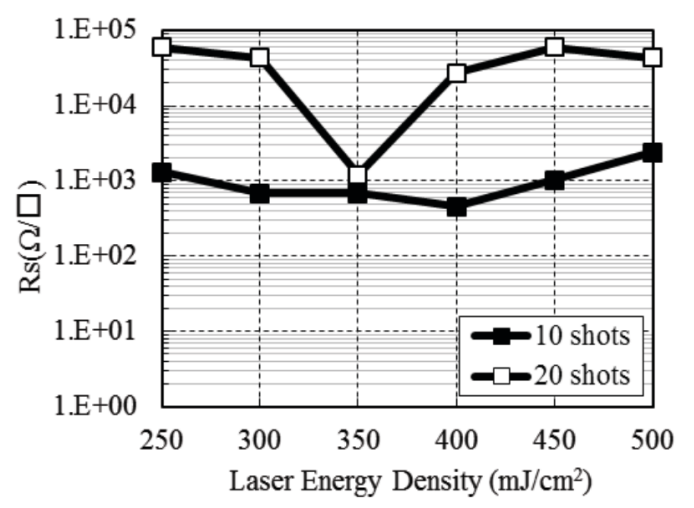

Figure 3. $R_{s}$ as a function of laser pulse shot number for laser annealed-only samples

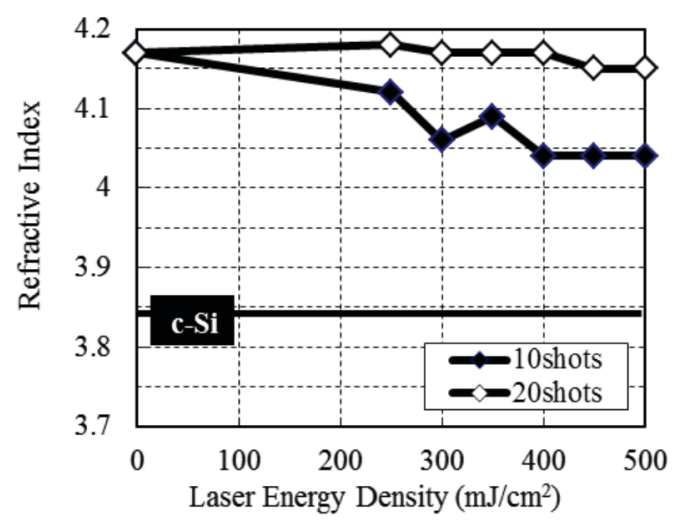

Figure 4.Refractive index of a-Si layer $\mathrm{x}$ as a function of laser pulse shot number for laser annealed-only samples

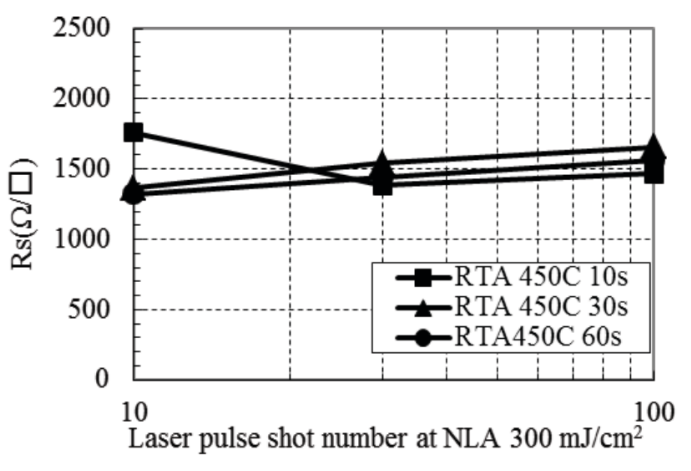

Figure 5. $R_{s}$ as a function of laser pulse shot number for samples subjected to RTA + NLA

\section{Summary}

We found that the value of sheet resistance was slightly increased with the laser pulse number in the samples subjected to preannealing RTA followed by NLA. This phenomenon is considered due to the interaction of dopant and supersaturated defect in the remaining a-Si layer results in the deactivation of the dopant. The optimumnumber of laser pulse shot is 10 shots. In addition, it is showed that NLA-only does not have enough energy to regrow a-Si layer and activate dopant layer, even when the pulse shot was increased.

\section{Acknowledgments}

We would like to thank Mr. T. Suzuki and Mr. T. Nakazawa for their help in preparing the samples. This work is supported by Ministry of Education Malaysia, UniversitiTeknologi Malaysia Research University Grant Scheme (PY/2014/03088) andin part by a Grantin-Aid for the Global Center of Excellence for HighLevel Global Cooperation for Leading-Edge Platform on Access Spaces from the Ministry of Education, Culture, Sport, Science, and Technology, Japan.

\section{References}

1. H.Dennard, F.H Gaensslen, H-N Yu, V.L. Rideout, E. Bassous, and A.R. Leblanc, IEEEJ. Solid-State Circuits, SC-9 (1974) p.256.

2. H.W. Kennel, M.D. Giles, M. Diebel, P.H. Keys, J. Hwang, S. Govindaraju, M. Liu, and A. Budrevich, in : The 14th IEEE Intl. Conf. on Adv. Thermal Proc. of Semiconductors - RTP 2006(2006)p.85.

3. T. Yamamoto, T. Kudo, T.Sukegawa, Y. Wang, L. Feng, S. Talwar, and M. Kase, Ext. Abs. of The 5th Workshop on Junction Technology 2005(2005)p.19.

4. M.C. Ozturk, J.J. Wortman, C.M. Osburn, A, Ajmera, G.A. Rozgonyi, E. Frey, W. Chu, and C. Lee, IEEE Transactions on Electron Devices 35 (1988)p.659.

5. S. Aronovitz, H. Puchner and J. Kimball, J. Appl. Phys. 85 (7) (1999) p. 3494.

6. S. Saito, K. Kumagai and T. Kondo, Appl. Phys. Lett. 63 (2) (1993)p. 197. 
7. S.R. Aid, S. Matsumoto, and G. Fuse, Phys. Status Solidi A 208(7) (2011) p.1646.

8. S.R. Aid, S. Matsumoto, G. Fuse, and S. Sakuragi, Phys. Status Solidi A 208(12) (2011) p.2772.

9. N. E. B. Cowern, D. Alquier, M. Omri, A. Claverie, and A. Nejim, Nucl. Inst. Meth. in Phys. Res. B. 148 (1999)p. 257.
10. S.R. Aid, S. Hara,Y.Shigenaga, T. Fukaya, Y.Tanaka,S. Matsumoto, G.Fuse, andS.Sakuragi, Jpn. J. Appl. Phys. 52 (2) (2013) p. 026501.

11. S. C. Jain, W. Schoenmaker, R. Lindsay, P. A. Stolk, S. Decoutere, M. Willander and H. E. Maes, J. Appl. Phys. 91 (2002)p. 8919. 